\title{
RITA Project: an Ambient Assisted Living solution for independent and safely living of aging population
}

Raffaele Esposito ${ }^{1}$, Manuele Bonaccorsi ${ }^{1}$, Dario Esposito ${ }^{1}$, Massimo Filippi ${ }^{1}$, Erika Rovini $^{1}$, Michela Aquilano ${ }^{1}$, Filippo Cavallo ${ }^{1}$, Paolo Dario ${ }^{1}$

\begin{abstract}
This paper presents the work carried out during the RITA Project, a study that focused on designing and implementing Ambient Assisted Living (AAL) services in the real context of Province of Pisa (Tuscany, Italy). The main objective of the RITA Project was to demonstrate the efficiency and the feasibility of new sociomedical services based on AAL approach. The user target of this project were elderly persons of Pisa area, living mainly alone or with a partner at their home, and their formal and informal caregivers. According to their needs new services and ICT system were developed and tested in order to improve the sense of safety of elderly people and caregivers.
\end{abstract}

\footnotetext{
${ }^{1}$ Raffaele Esposito, Manuele Bonaccorsi, Dario Esposito, Massimo Filippi, Erika Rovini, Dr. Michela Aquilano, Dr. Filippo Cavallo, Prof. Paolo Dario

The BioRobotics Institute, Scuola Superiore Sant'Anna, viale Rinaldo Piaggio 34, 56025 Pontedera (PI), Italy, e-mail: $\underline{\text { r.esposito@sssup.it, f.cavallo@sssup.it }}$
} 
Raffaele Esposito, Manuele Bonaccorsi, Dario Esposito, Massimo Filippi, Erika Rovini, Michela Aquilano, Filippo Cavallo, Paolo Dario

\section{Introduction}

People older than 65 years are the fastest growing segment of the European population and they will account for a third by 2060 [1]. As aged people, most of them are affected by physical and cognitive deficits and often need family or caregivers' support for their daily activities and health monitoring and care. For these reasons the ageing of the population is raising significant issues for new sustainable economic and welfare systems in order to meet needs of elderly citizens, families and caregivers in an adequate way and to impact positively on their quality of life and quality of work/services. In order to face the cost-effectiveness of new technologies for elderly three factors have to be considered:

- The increasing demand for elderly cares

In Europe people over 65 years will be a third of the population by 2060 and the consequence will be the increase of the demand for care due to a growing number of chronically ill people. The ageing of the society will led to an increasing demand for Nurse Practitioners (+94\% in 2025) [2] and Physician Assistants (+72\% in 2025) [3] with several implications for quality of care and for the configuration of future cost-effectiveness care delivery systems.

- The reduction of funds for social-medical services

The decreasing of worker population [4] and the reduction of funds for socialmedical services, due to the current economic crisis, couldn't fulfill the demand for care.

- The growth of smart technology market

In the last years we are witnessing from one side to the rapid increase of "intelligence" of technologies and at the same time to the improvement of their economic accessibility among common people (i.e. computers and smartphones). According to this phenomenon it is estimated that medical electronics equipment production will increase from the $\$ 91 \mathrm{Bn}$ in 2011 to the $\$ 119 \mathrm{Bn}$ in 2017 with an average rate of $4.6 \%$ per year [5]. Particularly the EU smart home market is estimated to grow from $\$ 1,544.3$ million in 2010 to $\$ 3,267$ million in 2015[6].

On the base of these premises the AAL services and the ICT systems could be a valid solution to support the independence of elderly people and improve a sustainable healthcare system.

In the last years, several research projects have been focused on new approaches and solutions for innovating socio-medical services, based on the use of ICT devices that could improve safety and independence of elderly persons. Some examples of these projects belong to the Ambient Assisted Living Joint Programme (AAL-JP): CARE [7], DOMEO [8], HOPES [9] and CARE@HOME [10Errore. L'origine riferimento non è stata trovata.] etc. Considering this context, the Tuscany Region (Italy) funded RITA Project to develop an 'aging-in-place' model, in which elderly 
people live as long as possible independently and safely in their own homes and caregivers can remotely follow them. RITA aims to demonstrate the potential efficiency of socio-medical services based on an Ambient Assisted Living (AAL) approach, the interest among elderly and caregivers and the sustainability of these AAL services in Tuscany context. So this project studied and designed services and ICT technologies suited for the Tuscany context and new legal framework needed to implement these new services in Tuscany. RITA Project was not only 'elderly people centered', but it also involved all persons supporting senior citizens, as relatives, caregivers, socio-medical workers and volunteers. This document reports an overview of the technological work carried out in RITA Project for designing and testing AAL services and ICT devices.

\section{Methods}

RITA consisted of two parts (see Figure 1):

Creative part included:

Phase 1: Analysis of (a) elderly people's needs and quality of life, and (b) attitude towards current and future technology.

Phase 2: Design of services and ICT systems.

Evaluation part included:

Phase 3 Assessment of developed services and ICT systems.

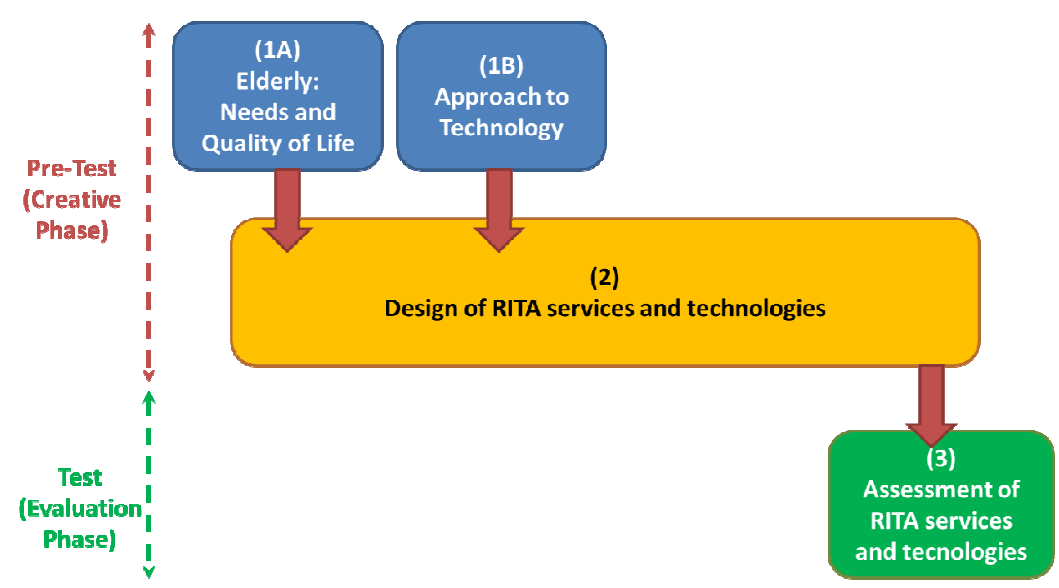

Figure 1. RITA Project: work phases 
Raffaele Esposito, Manuele Bonaccorsi, Dario Esposito, Massimo Filippi, Erika Rovini, Michela Aquilano, Filippo Cavallo, Paolo Dario

\subsubsection{Phase 1 (a) Elderly Needs and Quality of life}

During the Phase 1, researchers involved elderly citizens and caregivers living in the province of Pisa, Italy, through a number of focus groups and interviews in ten local end-user organizations and in some cases at elderly homes. Ad-hoc questionnaires, combining multiple choice and open questions, were used to define the profile of older persons and identify their perceived needs and quality of life. The interviewees were 211 elderly people $65+$ without severe cognitive pathologies and 68 formal and informal caregivers. The details of participants are showed in Table I and Table II.

Table I Elderly people's characteristics

\begin{tabular}{ccccc}
\hline \multicolumn{5}{c}{ Number of Elderly } \\
\hline Gender & $\mathbf{6 5 - 7 5}$ Years & Over 75 Years & Unspecified age & TOT \\
Men & 24 & 24 & 4 & $\mathbf{5 2}$ \\
Women & 36 & 120 & 3 & $\mathbf{1 5 9}$ \\
TOT. & $\mathbf{6 0}$ & $\mathbf{1 4 4}$ & $\mathbf{2 1 1}$ \\
\hline Gender & Min. of Elderly & SD \\
Men & 65 & Max & Mean & 9 \\
Women & 65 & 100 & 76 & 8 \\
\hline
\end{tabular}

Table II Caregivers' characteristics

\begin{tabular}{c|cccc|ccc}
\hline & \multicolumn{4}{|c|}{ Formal Caregivers } & \multicolumn{3}{c}{ Informal Caregivers } \\
\hline \multirow{2}{*}{ Gender } & \multicolumn{2}{c}{ Men } & Women & TOT & Men & Women & TOT \\
& \multicolumn{2}{|c}{22} & 33 & 55 & 5 & 8 & 16 \\
\hline \multirow{2}{*}{ Role } & Health Workers & \multicolumn{2}{c|}{ Volunteers } & Family & \multicolumn{2}{c}{ Private Care } \\
& \multicolumn{2}{c}{23} & \multicolumn{2}{c}{32} & 13 & 0 & \\
\hline \multirow{2}{*}{ Age } & $\mathbf{0 - 4 0}$ & $\mathbf{4 1 - 6 0}$ & $\mathbf{6 0 +}$ & ND & $\mathbf{0 - 4 0}$ & $\mathbf{4 1 - 6 0}$ & $\mathbf{6 0 +}$ \\
& 12 & 22 & 16 & 5 & 0 & 2 & 11 \\
\hline
\end{tabular}

In general elderly users were enough satisfied about their life and their current independence; however the main aspects influencing their perception of quality of life concerned possible degeneration of their health status, safety and social life.

In summary from these surveys three main elderly requirements emerged:

1. increasing the attention of doctors and caregivers about their health;

2. living at their own home maintaining their autonomy and independence but in a safe and secure context; 
RITA Project: independent and safely living of aging population

3. carrying out social activities to reduce their sense of loneliness and general negative feelings.

Looking to caregivers point of view, two main critical points were highlighted:

1. the willing to follow more carefully the elderly without increasing their burden and having a negative impact on their quality of life;

2. the hope that technologies could help them to monitor old persons, especially during their absence, and inform them in case of possible dangerous events.

Caregivers felt that assisting elderly persons was a heavy work and its burden influenced negatively their life. At the same time, they would like to follow more efficiently the older users and for this reason they believed that current technologies could help them to assist elderly relative.

\subsubsection{Phase 1 (b) Elderly and Technology}

Fifty-three (53) older adults drew up a specific questionnaire in order to investigate their attitude about current and future technology in the context of their home, daily life, free time and healthcare. This survey showed that participants used different kind of technology; particularly they were familiar with home appliance and electronic devices for everyday use. Interestingly elderly people involved in the study declared to have a mobile phone to dial and answer call, even if only few of them were able to send SMS. Also most of them used technological devices for monitoring their health status, such as blood pressure or glycaemia, but few of them used sensor devices for monitoring the domestic environment and even less used a tele-assistance system.

About attitude towards future technology, most of elderly people reported to be interested in using technology for monitoring their health status and for controlling remotely their home when they were both inside and outside of it. Also they thought that internet and informatics were useful to keep relationship with family and friends. At last the half of the sample emphasized the benefit of a tele-gym system in keeping a good fitness.

\subsection{Phase 2 Design of Services and ICT System}

The new socio-medical services based on AAL approach may allow elderly people to stay in their own homes for as long as possible delaying institutional care. 
Raffaele Esposito, Manuele Bonaccorsi, Dario Esposito, Massimo Filippi, Erika Rovini, Michela Aquilano, Filippo Cavallo, Paolo Dario

Many studies and technologies [11] that can assist older adults to continue living at home with safety and independence were developed, but few of them faced the cost of the new technologies. Suryadevare and Mukhopadhyay [12] developed a wireless sensor network integrated with ZigBee modules for monitoring ADLs of elderly living alone reporting that it was a "low cost" solution. Also Lotfi et al. [13] implemented a "low cost" sensor network including movement and door entry point sensors for supporting independent living of the elderly suffering from dementia. Tomita et al. [14] conducted a study with frail elderly people, who lived alone, based on a computer with Internet access and X10-based smart home technology while Van Hoof et al [15] used a prototype of the Unattended Autonomous Surveillance system to promote the ageing in place.

In RITA Project in order to meet elderly people's need a modular and pervasive wireless sensor network was developed. Furthermore an Ambient Intelligence (AmI) Infrastructure was created for the integration of information collected from all sensors.

The modules (Figure 2) and services implemented are described below.

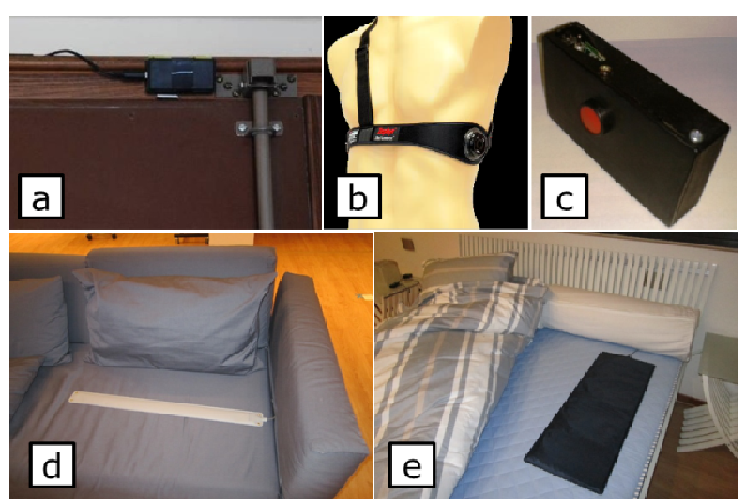

Figure 2. Modules developed for RITA Project: (a) system for door monitoring; (b) commercial band monitoring physiological signals (BioHarness ${ }^{\mathrm{TM}}$, Zephyr); (c) localization and alarm module; (d) sofa monitoring (BOS, Recora); (e) bed monitoring (COS, Recora)

General health status monitoring: the commercial wearable monitoring system BioHarness ${ }^{\mathrm{TM}}$ (Zephyr) (Figure 2.b) was used to measure physiological parameters as heart rate, respiration rate, temperature, activity and posture. The system was composed by an electronics module and an ad-hoc wearable band. Data could be transmitted directly to a PC, via Bluetooth, and viewed in real- time or logged inside the electronic module and later uploaded and transmitted to clinicians. In this way physiological data could be analyzed and monitored remotely by doctors and so elderly people could receive more attention about their health by clinicians also beyond direct medical examinations. 
RITA Project: independent and safely living of aging population

Indoor and outdoor localization: a wearable module (Figure 2.c), powered by a Lithium battery, was developed for localizing elderly and monitoring their motor activity in daily life. The system was composed by an inertial sensor (iNEMO-M1, STMicroelectronics) to monitor motor activity, a GPS receiver and GSM/GPRS module for outdoor localization (GM862-GPS, Telit), a ZigBee module (SPZB250M, STMicroelectronics) for indoor localization and the GPS and ZigBee antennas. The system was able to switch automatically from GPS (outdoor open space) to ZigBee module (indoor environment). This system could help caregivers to know always where old persons were especially during their absence. Furthermore the wearable module allowed elderly people, that have feelings of vulnerability and insecurity, to go out for their activities in safety because they knew that they could be localized with high precision in case of need.

Help request: the wearable module (Figure 2.c) was equipped with a red button that could be pushed by elderly people in case of need. Thanks to the GSM/GPRS module the caregivers received an SMS help request that informed if the elderly person was inside or outside home. In this way elderly people could maintain their autonomy and independence and the caregivers could be dismissed from a permanent assistance.

Domestic environment monitoring: a wireless indoor sensor network based on ZigBee technology was implemented and some environmental sensors were integrated into domestic spaces. In particular sensors acquired information about door opening/closing (Figure 2.a), user presence on sofa (Figure 2.d) and user presence in bed (Figure 2.e). All these modules were coordinated and managed by a PC, present inside the house and working as server. This sensor network allowed elderly people to live at home in security because unexpected environmental changes were detected and alert requests were promptly sent to the caregiver. Furthermore these sensors together with the localization module allowed to estimate elderly motor and static activities (for example, time in front TV) and recognise some activities as sleeping or napping. This information could be useful for caregivers to plan activities to maintain senior users active from the motor and social points of view.

Remote control interfaces: ad-hoc computer interfaces for the indoor environment monitoring and outdoor localization were developed. These components were conceived to allow caregivers to easily control elderly status. Also some information as help request, localization and door status were transmitted on caregiver mobile phone through SMS. Finally a mobile phone interface for physiological parameters was developed. 
Raffaele Esposito, Manuele Bonaccorsi, Dario Esposito, Massimo Filippi, Erika Rovini, Michela Aquilano, Filippo Cavallo, Paolo Dario

\subsection{Phase 3 Experimentation of Services and ICT system}

Two experimentation loops were conducted with elderly people and formal caregivers for testing RITA services and ICT system. The first loop was organized at DomoCasa Lab in Peccioli (Pisa, Italy) and involved 17 persons over 65 years old. During the test the elderly volunteers tested RITA sensors and services, described in the previous paragraphs, and then reported their feedback through a questionnaire. Data suggested that the minority of the sample thought that using the showed technology and services could have an excessively negative impact on their lifestyle, while the majority of them perceived the benefit of this technology use in everyday. At last elderly people reported that the perceived sense of safety and quality of life could improve (Figure 3) thanks to the technology and services use.

$=$ Under75 = 00er75

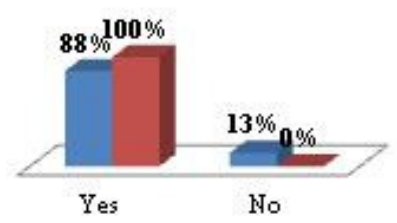

Figure3: Do you think that the use of RITA services and ICT system in everyday life could improve your quality of life?

The second loop was conducted at a residential care home for elderly with 9 formal caregivers and it unfolded with the same modalities of the first. All participants reported that the elderly people's quality of life could improve if this technology and services were implemented in everyday life. Also the caregivers thought that showed technology would increase the quality of job of socio-medical workers (Figure4 a) and only a few of them suggested that all this would lead to a less personal relationship between caregiver and elderly person (Figure4 b).
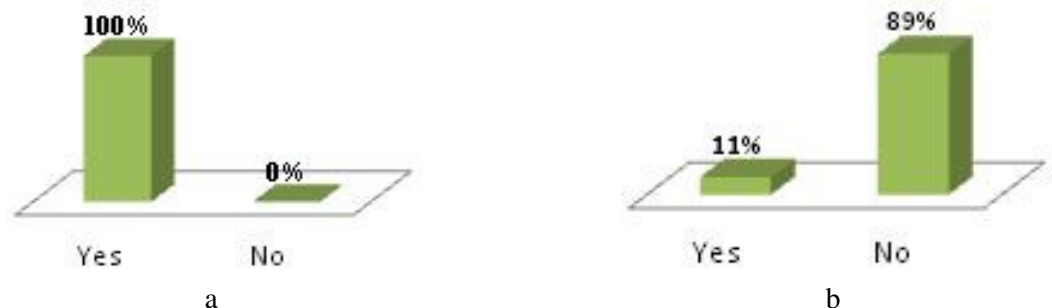

Figure4 a: Do you think that the use of RITA services and ICT system in everyday life could increase the quality of job of socio-medical workers?

b: Do you think that the use of RITA services and ICT system could lead to a less personal relationship between caregiver and elderly person? 
RITA Project: independent and safely living of aging population

\section{Conclusions}

As demonstrated in other studies conducted in Europe even in Tuscany, a new sustainable economic and welfare system to develop an aging in place model is indispensable.

The feasibility and utilization of new technologies for elderly is related to the perception of benefits due to their use, but the budget is also not a negligible aspect. Even if the cost-effectiveness was difficult to elicit, promoting ageing in place and facilitating older people to live independently in their homes should lead to more effective forms of care for elderly. In fact the new technologies, home and health monitoring, could reduce the burden on informal and formal caregivers. Thanks to a new socio-medical services based on AAL approach the informal caregivers could be always informed about their relative's condition reducing the necessity of their presence at home of their older relatives; this condition allows them to reduce costs due to time savings and avoidance of travelling and also to avoid to take frequently day or time off work for older persons' cares. Also the formal caregivers could be supported in their caring work offering a more focused and efficient job that could allow to caregivers to spent more time for a direct contact with elderly people.

In line with these objectives of efficient cares and with the AAL approach the RITA Project designed some possible socio-medical services, starting from the analysis of elderly persons' real needs, that are based on the use of "low-cost" ICT system. This research obtained positive and encouraging results, in fact, at the end of the project old people involved in the study said that they were interested in using the RITA technology and services in everyday life because this technology could improve the their perceived safety feeling and quality of life, not having a negative impact on their lifestyle and home environment. Moreover also the caregivers asserted that RITA services could improve the quality of job of socio-medical workers without damaging the personal relationship between caregiver and elderly assisted person.

In conclusion, RITA Project showed that a care model, in which elderly people live independently and safely in their own homes as long as possible, is feasible. Achieved results and feedbacks suggested that both elderly people and caregivers are favorably disposed to use new services and ICT systems in everyday life, if they perceive the benefits, related to the technology use, on the elderly people's quality of life and the quality of welfare services offered by social-medical workers and families. 
Raffaele Esposito, Manuele Bonaccorsi, Dario Esposito, Massimo Filippi, Erika Rovini, Michela Aquilano, Filippo Cavallo, Paolo Dario

\section{Acknowledgements}

The RITA project has been supported by Regione Toscana under the POR CReO FESR 2007-2013 Programme, Asse 1 Attività 1.1 Linea di intervento D. Furthermore authors thank Società della Salute -Area pisana - which provided contacts with local associations and service providers, working with elderly people, allowing the involvement of a large number of elderly persons and caregivers in the project.

\section{References}

1. European Commission (DG ECFIN) and Economic Policy Committee (AWG) (2012) The 2012 Ageing Report: Economic and budgetary projections for the EU27 Member States (2010-2060). European Economy 2/2012.

2. D. I. Auerbach, "Will the NP Workforce Grow in the Future?: New Forecasts and Implications for Healthcare Delivery," Medical Care, vol. 7, no. 7, July 2012.

3. R. S. Hooker, J. F. Cawley and C. M. Everett, "Predictive Modeling the Physician Assistant Supply: 2010-2025," Public Health Reports, vol. 126, pp. 708-716, September 2011.

4. Eurostat, "European social statistics - edition 2013," 2013.

5. iNEMI Advancing manufacturing technology, "iNEMI Technology Roadmap 2013," 2013.

6. Markets and Markets, "European Smart Homes and Assisted Living Market (2010 - 2015)," 2011.

7. Belbachir A N, Litzenberger M, Schraml S, Hofstatter M, Bauer D, Schon P, Humenberger M, Sulzbachner C, Lunden T, Merne M (2012) CARE: A dynamic stereo vision sensor system for fall detection. Proc. IEEE ISCAS2012, doi: 10.1109/ISCAS.2012.6272141.

8. Rumeau P, Vigouroux N, Boudet B, Lepicard G (2012) Home deployment of a doubt removal telecare service for cognitively impaired elderly people: A field deployment. Proc. IEEE CogInfoCom 2012, doi: 10.1109/CogInfoCom.2012.6422015.

9. Resca A, Spagnoletti P (2011) ICT and elderly: a tool for supporting on line communities or off line communities? Proc. Personal Health Records and patient-oriented infrastructures.

10. Pommeranz A, Fitrianie S, Alers H, Guldemond N (2012) Care@ Home: An integrated approach to care and social inclusion of elderly. AAL Forum 2012.

11. Morris et al. (2013) Smart-Home Technologies to Assist Older People to Live Well at Home,Journal of Aging Science 2013

12. Suryadevara NK, Mukhopadhyay SC (2012) Wireless sensor network based home monitoring system for wellness determination of elderly. IEEE Sensors Journal 12: 1965-1972.

13. Lotfi A, Langensiepen C, Mahmoud SM, Akhlaghinia MJ (2010) Smart homes for the elderly dementia sufferers: Identification and prediction of abnormal behaviour. J Ambient Intell Humaniz Comput.

14. Tomita MR, Mann WC, Stanton K, Tomita AD, Vidyalakshmi S (2007) Use of currently available smart home technology by frail elders: process and outcomes. Top Geriatr Rehabil 23: 24-34

15. van Hoof J, Kort HS, Rutten PG, Duijnstee MS (2011) Ageing-in-place with the use of ambient intelligence technology: Perspectives of older users. Int J Med Inform 80: 310-331. 